\title{
Endogenous cGMP-dependent protein kinase reverses EGF-induced MAPK/ERK signal transduction through phosphorylation of VASP at Ser239
}

\author{
YAN TAO $^{1}$, YIN-JIE GU ${ }^{2}$, ZHI-HONG CAO ${ }^{2}$, XIU-JUAN BIAN ${ }^{3}$, TING LAN $^{1}$, JIAN-RONG SANG ${ }^{1}$, \\ LU JIANG $^{1}$, YING WANG ${ }^{1}$, HAI QIAN ${ }^{1}$ and YONG-CHANG CHEN ${ }^{1}$ \\ ${ }^{1}$ School of Medical Science and Laboratory Medicine, Jiangsu University, Zhenjiang, Jiangsu 212013; \\ ${ }^{2}$ Intensive Care Unit, Affiliated Hospital of Jiangsu University, Yixing, Jiangsu 214200; \\ ${ }^{3}$ Department of Respiratory Diseases, Affiliated Hospital of Jiangsu University, Zhenjiang, Jiangsu 212001, P.R. China
}

Received April 24, 2012; Accepted July 25, 2012

DOI: $10.3892 / \mathrm{ol} .2012 .851$

\begin{abstract}
In our previous study, we demonstrated that type II cGMP-dependent protein kinase (PKG II) was expressed at lower levels in different human cancer cell lines and that exogenous PKG II inhibited epidermal growth factor (EGF)-induced MAPK/ERK signaling. In order to investigate its functions further in this signaling pathway, it is necessary to elucidate whether endogenous PKG has the same effect or not. This study aimed to investigate the possible inhibitory effect of endogenous PKG activity on EGF-induced MAPK/ERK signal transduction in human lung cancer cells and its mechanism. Human small cell lung carcinoma cells (SCLCs) were treated with the PKG-selective cGMP analog 8-pCPT-cGMP to activate endogenous PKG, EGF and cGMP followed by EGF, respectively. The results showed that increased endogenous PKG activity inhibited the EGF-induced phosphorylation of the epidermal growth factor receptor (EGFR) and the binding between Sos1 and Grb2. In addition, EGF-triggered Ras activation was reversed by increased endogenous PKG activity. While the EGF-induced phosphorylation of MEK and ERK were inhibited by increased endogenous PKG activity, there was a significant increase of phosphorylated vasodilator-stimulated phosphoprotein (p-VASP) at Ser239. Furthermore, we investigated whether endogenous PKG exerted its effects on EGF-induced MAPK/ERK signaling through phosphorylation of VASP at Ser239. Downregulation of the levels of p-VASP Ser239 by point mutation blocked the effects of endogenous PKG on EGF-induced MAPK/ERK signal transduction. The data shown here suggest that endogenous PKG reverses the
\end{abstract}

Correspondence to: Dr Yan Tao, School of Medical Science and Laboratory Medicine, Jiangsu University, 301 Xue-fu Road, Zhenjiang, Jiangsu 212013, P.R. China

E-mail: yantao1981@126.com

Key words: endogenous, cGMP-dependent protein kinase, VASP, MAPK/ERK, small cell lung carcinoma
EGF-induced MAPK/ERK signaling pathway by phosphorylating VASP at Ser239.

\section{Introduction}

Epidermal growth factor receptor (EGFR) is a $170 \mathrm{kDa}$ transmembrane tyrosine kinase that belongs to the erbB family of receptor tyrosine kinases and contains three domains: an extracellular domain, a cross-membrane domain and an intracellular domain. Further, the intracellular domain can be divided into three sub-domains: the approximate membrane, tyrosine kinase and C-terminal sub-domain (1). EGFR has been strongly implicated in the biology of human epithelial malignancies, with therapeutic applications in cancers of the colon, head and neck, lung and pancreas (2). Its mechanism of activation relies on receptor dimerization and auto-phosphorylation (3). The activation of EGFR triggers several signal transduction pathways, including the MAPK-mediated signaling pathway (4). Activated EGFR promotes the binding between adapter protein Grb2 and Sos1 (5). The activated Sos1 can lead to the activation of small $\mathrm{G}$ protein Ras. Ras proteins act as molecular switches that alternate between inactive GDP-bound and active GTP-bound states. The small GTPase Ras has a prominent role in the signaling pathways leading from activated growth factor receptors to ERKs (6-8).

cGMP-dependent protein kinases (PKGs) are serine/threonine kinases. Two main classes of PKG have been indentified: type I PKG (PKG I) and type II PKG (PKG II) $(9,10)$. PKG II is a membrane-bound enzyme primarily found in the epithelial cells of the intestine (11). PKG phosphorylates target effectors, including vasodilator-stimulated phosphoprotein (VASP), inositol-1,4,5-trisphosphate (IP3) receptor-associated cGMP kinase substrate (IRAG) and thromboxane A2 (TXA2) receptor (12-15). Recent research has revealed that PKGs were associated with the proliferation of tumor cells (16). Recently, our experiments indicated that increased exogenous PKG II activity inhibited the proliferation of gastric cancer cells (17). Our further study showed that the inhibitory effects of exogenous PKG II on EGF-triggered proliferation were associated with its effects on the MAPK/ERK signal trans- 
duction pathway (18). In light of this, we carried out further experiments to elucidate whether the endogenous PKG activity is able to reverse the EGF-induced MAPK/ERK signaling pathway, and to investigate its possible mechanism.

\section{Materials and methods}

Cell line. The human small cell lung carcinoma (SCLC) cell line was provided by the Institute of Cell Biology (Shanghai, China). The study was approved by the ethics committee of Jiangsu University, Jiangsu, China.

Reagents. Antibodies against p-MEK (Ser217/221) and p-EGFR (Tyr1068) were purchased from Cell Signaling Technology, Inc. (Danvers, MA, USA). Antibodies against pan-Ras, Sos1, Grb2, VASP and p-VASP (Ser239) were from Santa Cruz Biotechnology, Inc. (Santa Cruz, CA, USA). Antibodies against pERK1/2 (Thr202/Tyr204) and GAPDH were from Bioworld Technology Co., Ltd. (St. Louis Park, MN, USA). Horseradish peroxidase (HRP)-conjugated secondary antibodies were from Jackson ImmunoResearch Laboratories, Inc. (West Grove, PA, USA). The cellular permeable cGMP analog 8-pCPT-cGMP was from Calbiochem (San Diego, CA, USA). Electrochemiluminescence (ECL) reagent was from Millipore (Billerica, MA, USA). Dulbecco's modified Eagle's medium (DMEM) and new-born calf serum (NBCS) were from Gibco (Invitrogen Life Technologies, Grand Island, NY, USA).

Cloning and transfection. As described by Geese et al, mutagenesis of the VASP phosphorylation site Ser239 (S239A) was performed using the QuickChange site-directed mutagenesis kit (Stratagene, La Jolla, CA, USA) according to the manufacturer's instructions (18). The primers used for pMSCV+EGFP-VASP SAT S239A were CTCAGGAAAGTCGCCAAGCAGGAGGAG-GCC (forward) and GGCCTCCTCCTGCTTGGCGACTTT CCT-GAG (reverse). A BamHI restriction site was introduced into the 5-end of the S239A-S primer, and an EcoRI restriction site was introduced into the 5-end of the S239A-A primer. The S239A fragment was then subcloned into lentiviral transfer vector FUGW (F, HIV-1 flap sequence; U, human polyubiquitin promoter; G, green fluorescent protein; W, woodchuck hepatitis virus post-transcriptional regulatory element) by Bam HI and EcoRI double digestion. Recombinant lentiviral particles were produced in $293 \mathrm{~T}$ cells by transient cotransfection involving a three-plasmid expression system (20), and cells were transfected as described previously (21). The expression levels of p-VASP Ser239 were verified by western blotting analysis.

Ras activity assay. The activity of Ras was detected with the pull-down method as described previously (22). In brief, cells growing on $100-\mathrm{mm}$ culture plates were washed three times with cold phosphate-buffered saline (PBS) and lysed by adding $400 \mu 1$ of the lysis buffer [25 mM HEPES (pH 7.5), $150 \mathrm{mM}$ $\mathrm{NaCl}, 1 \%$ NP40, 10\% glycerol, $25 \mathrm{mM} \mathrm{NaF}, 10 \mathrm{mM} \mathrm{MgCl}$, $0.25 \%$ sodium deoxycholate, $1 \mathrm{mM}$ EDTA, $1 \mathrm{mM} \mathrm{Na} \mathrm{VO}_{4}$, $10 \mu \mathrm{g} / \mathrm{ml}$ aprotinin and $10 \mu \mathrm{g} / \mathrm{ml}$ leupeptin]. The sample was collected and centrifuged $\left(14,000 \times \mathrm{g}, 4^{\circ} \mathrm{C}, 10 \mathrm{~min}\right)$ to get rid of the debris. The supernatant was incubated with glutathione sepharose beads and glutathione S-transferase-Ras-RBD (GST-RBD) at $4^{\circ} \mathrm{C}$ for $1 \mathrm{~h}$. The beads were washed 3 times with lysis buffer and heated in boiled water to release proteins. The protein samples (containing active Ras) were analyzed by western blotting with an antibody against pan-Ras.

Co-immunoprecipitation. The cells growing on the $100-\mathrm{mm}$ culture plate were washed twice with cold PBS and lysed by adding $1 \mathrm{ml}$ RIPA buffer [50 mM Tris- $\mathrm{HCl}$ ( $\mathrm{pH} 7.4), 1 \%$ Triton X-100, 1 mM EDTA, $1 \mathrm{mM}$ leupeptin, $1 \mathrm{mM}$ phenylmethylsulfonyl fluoride, $10 \mathrm{mM} \mathrm{NaF}, 1 \mathrm{mM} \mathrm{Na}_{3} \mathrm{VO}_{4}$ ) per plate. Antibodies against Grb2 and Sos1 and isotype matched IgG were used for immunoprecipitation.

Western blot assay. Sample proteins were separated on SDS-PAGE gels and blotted onto polyvinyl difluoride (PVDF) membrane. The PVDF membrane was blocked with $3 \%$ (w/v) bovine serum albumin (BSA) in TBS-T for $1 \mathrm{~h}$ at room temperature. The incubation with the primary antibody was at $4^{\circ} \mathrm{C}$ overnight, and with the secondary antibody was $1 \mathrm{~h}$ at room temperature, with three washes after each incubation. Electrochemiluminescence reagents were used to show the positive bands on the membrane. The bands were detected by Typhoon 9400 (GE Healthcare, Waukesha, WI, USA).

\section{Results}

Increased endogenous PKG activity reverses EGF-induced Tyr1068 phosphorylation of EGFR in SCLC cells. Stimulation of EGFR by its natural ligand EGF causes the dimerization and the auto-phosphorylation of tyrosine of the receptors. Tyrosine 1068 (Y1068) is one of the auto-phosphorylation sites of EGFR. Phosphorylation of this site is associated with MAPK-mediated signaling. In this study, western blot assay was applied to investigate the effect of endogenous PKG activity on the phosphorylation of EGFR (Y1068) in EGF-treated SCLC cells. Results showed that EGF treatment notably increased the EGFR phosphorylation at Y1068. In cells with the treatment of cGMP followed by EGF, this phosphorylation was significantly decreased compared with the EGF treatment alone (Fig. 1). This demonstrates that increased endogenous PKG activity inhibited the Y1068 phosphorylation of EGFR induced by EGF.

Increased endogenous $P K G$ activity prevents binding between adaptor protein Grb2 and Sos1 in SCLC cells. The phosphorylated tyrosines on EGFR provide binding sites for adaptor proteins. Among them, phosphorylated Tyr1068 is associated with the activation of the MAPK-mediated signaling pathway. Grb2 is a growth factor receptor-bound protein and Sos1 is a guanine nucleotide exchange factor (GEF). They play a role as adaptor proteins in the MAPK-mediated signaling pathway. The bind between them activates the downstream signal component Ras. Co-immunoprecipitation assay was applied to detect the binding between Grb2 and Sos1. Results showed that with the treatment of EGF, this binding between Grb2 and Sos1 increased. In the cells stimulated with cGMP followed by EGF, this binding decreased more than in cells with treatment of EGF only (Fig. 2). These results revealed that increased 


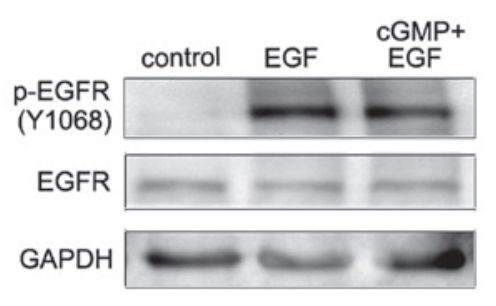

Figure 1. Endogenous PKG inhibits EGF-induced Y1068 phosphorylation of EGFR. Small cell lung cancer cells were serum-starved for $12 \mathrm{~h}$ and incubated with EGF $(100 \mathrm{ng} / \mathrm{ml})$ for $5 \mathrm{~min}$ or treated with 8-pCPT-cGMP $(250 \mu \mathrm{M})$ for $1 \mathrm{~h}$ and then with EGF $(100 \mu \mathrm{g} / \mathrm{ml})$ for $5 \mathrm{~min}$. Cells were harvested and subjected to western blotting. Results showed that EGF treatment induced an increase in the Y1068 phosphorylation of EGFR. cGMP treatment efficiently reversed the EGF-triggered Y1068 phosphorylation of EGFR. Results shown are representative of three separate experiments.

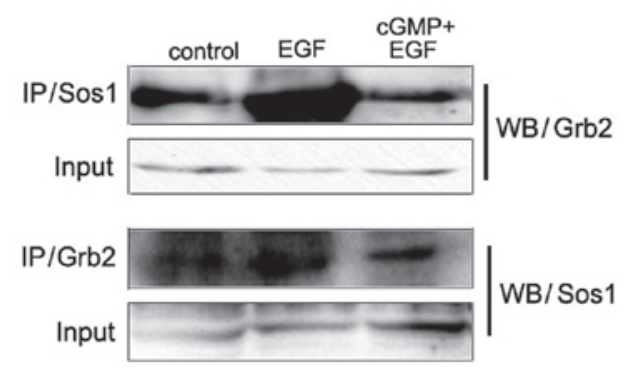

Figure 2. Endogenous PKG prevents EGF-induced binding between Sos1 and Grb2. Small cell lung cancer cells were serum-starved for $12 \mathrm{~h}$ and incubated with EGF $(100 \mathrm{ng} / \mathrm{ml})$ for $5 \mathrm{~min}$ or treated with 8-pCPT-cGMP $(250 \mu \mathrm{M})$ for $1 \mathrm{~h}$ and then with EGF $(100 \mathrm{ng} / \mathrm{ml})$ for $5 \mathrm{~min}$. Co-immunoprecipitation was performed to analyze the effect of EGF and endogenous PKG on the binding between Grb2 and Sos1. Results showed that with EGF treatment, the binding level between Grb2 and Sos1 was much higher than that in untreated cells. cGMP treatment significantly inhibited this binding. Results shown are representative of three separate experiments.

endogenous PKG activity inhibited the EGF-induced binding between Grb2 and Sos1.

Increased endogenous PKG activity inhibits EGF-activated Ras and the phosphorylation of MEK and ERK in SCLC cells. Ras is the key component in the MAPK-mediated signaling pathway. Once Ras is in GTP-bound form, it binds and activates Raf- 1 and starts the consequent activations of the serine/threonine kinase in the signaling pathway. Raf-1 is a regulator upstream of ERK in MAPK-mediated signal transduction pathway. Activated Raf-1 activates MEK by inducing its phosphorylation of two serine residues at positions 217 and 221. MEK1 and MEK2 are members of the dual specificity protein kinase family, which acts as a MAPK or ERK kinase. Pull-down assay and western blotting were applied to analyze the Ras activity induced by EGF or the combination of cGMP with EGF. Results showed that EGF activates Ras, while the later treatment reverses its effect on Ras activity (Fig. 3A). Furthermore, western blot analysis was applied to detect the phosphorylation of MEK and ERK induced by EGF or the combination of cGMP with EGF. Results indicated that with treatment of EGF alone, the phosphorylation level of both MEK and ERK increased, while with the treatments of cGMP followed by EGF, the phosphorylation level of MEK and ERK

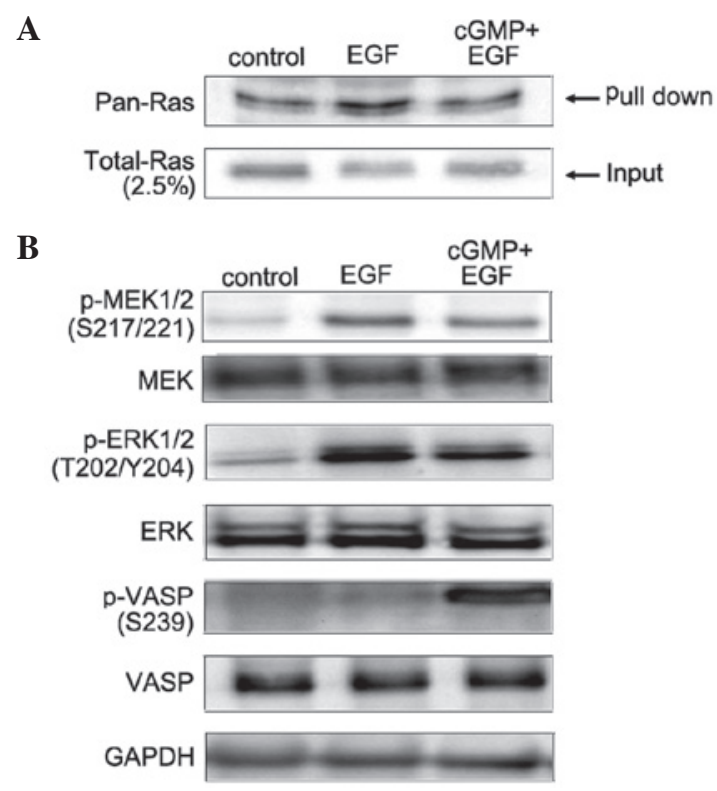

Figure 3. Endogenous PKG inhibits EGF-triggered Ras activation and the phosphorylation of MEK and ERK. Small cell lung cancer cells were serum -starved for $12 \mathrm{~h}$ and incubated with EGF $(100 \mathrm{ng} / \mathrm{ml})$ for $5 \mathrm{~min}$ or treated with 8-pCPT-cGMP $(250 \mu \mathrm{M})$ for $1 \mathrm{~h}$ and then with EGF $(100 \mathrm{ng} / \mathrm{ml})$ for 5 min. (A) Pull-down assay was applied to detect the activated Ras Cell lysate was prepared and equal amounts of protein were incubated with GST-RBD beads as described in Materials and methods. Binding complexes were collected by centrifugation, resolved by SDS-PAGE, and probed with anti-pan Ras antibody. Results showed that EGF treatment caused a notable activation of Ras. cGMP treatment inhibited EGF-induced Ras activation. (B) Cells were harvested and subjected to western blotting. Results showed EGF treatment induced an increase in the phosphorylation of MEK and ERK. cGMP treatment efficiently reversed the EGF-triggered phosphorylation of MEK and ERK, and caused a significant increase of p-VASP Ser239. Results shown are representative of three separate experiments.

notably decreased compared with EGF treatment, and there was a significant increase in the phosphorylation level of VASP at Ser239 (Fig. 3B). These results demonstrate that increased endogenous PKG activity prevents EGF-activated Ras and the phosphorylation of MEK and ERK in SCLC cells.

Downregulating the level of p-VASP Ser239 blocks the effects of endogenous PKG on EGF-induced MAPK/ERK signal transduction. VASP is known to be a substrate for several protein kinases in a variety of cells, including PKG (23). VASP harbors three phosphorylation sites: Ser157, located N-terminally to the central proline-rich region (PRR); and Ser239 and Tyr278, located in the Ena/VASP homology domain 2 (EVH2 domain) (24-26). Since p-VASP Ser239 increased while the phosphorylation level of MEK and ERK decreased in cells treated with cGMP followed by EGF, it was important to investigate whether endogenous PKG exerted its effects on the EGF-induced MAPK/ERK signaling pathway through phosphorylating VASP at Ser239. For the present study, we designed lentivirus-mediated EGFP-VASP Ser239 site mutation (239A) to block its phosphorylation in SCLC cells. The expression of EGFP (green) was used to present the positive infected cells, the infected efficiency was more than 95\% (Fig. 4A), and cells stably expressing 239A were named SCLC-239A. Furthermore, western blotting was applied to 
A

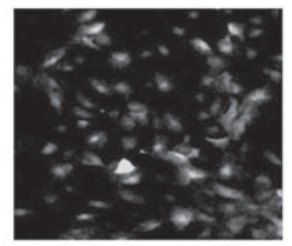

B

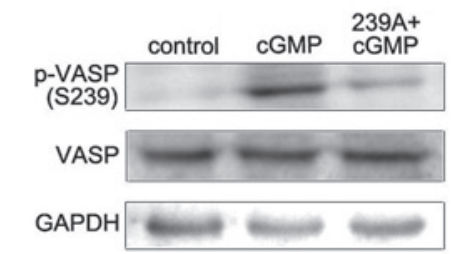

C
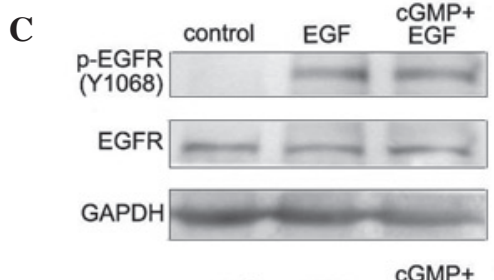

D

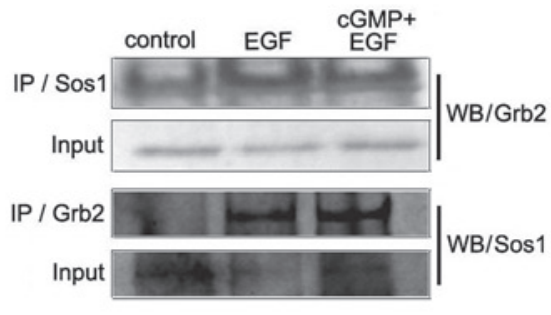

E
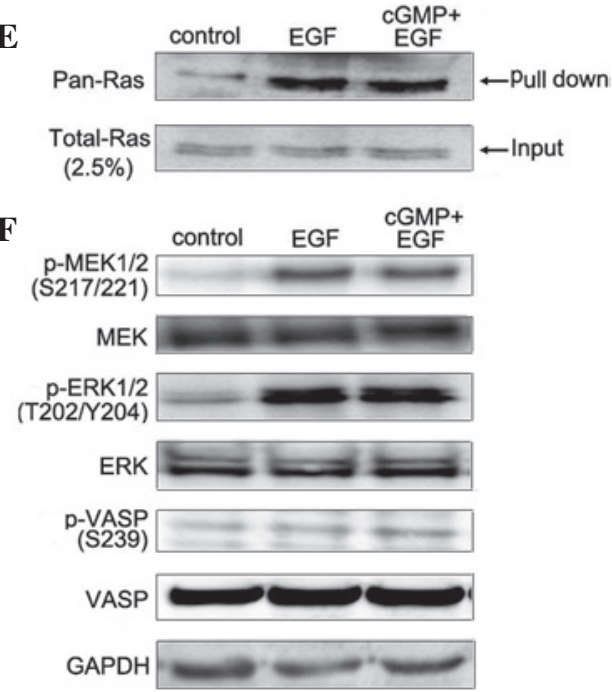

Figure 4. Blocking the phosphorylation of p-VASP S239 attenuates the effects of endogenous PKG on EGF-induced MAPK/ERK signal transduction. Small cell lung cancer cells infected with the lentivirus-mediated EGFP-VASP Ser239 site mutation (239A) were named SCLC-239A. (A) The infected efficiency was indicated by the expression of EGFP (green). (B) SCLC-239A cells were serum-starved for $12 \mathrm{~h}$ and treated with 8-pCPT-cGMP $(250 \mu \mathrm{M})$ for $1 \mathrm{~h}$. Cells were harvested and subjected to western blotting. Results showed that cGMP treatment did not increase p-VASP S239. SCLC-239A were serumstarved for $12 \mathrm{~h}$ and incubated with EGF $(100 \mathrm{ng} / \mathrm{ml})$ for $5 \mathrm{~min}$ or treated with 8-pCPT-cGMP $(250 \mu \mathrm{M})$ for $1 \mathrm{~h}$ and then with EGF $(100 \mathrm{ng} / \mathrm{ml})$ for $5 \mathrm{~min}$. Cells were harvested and subjected to western blotting. (C and F) Results showed that EGF treatment induced an increase in the phosphorylation of EGFR, MEK and ERK. cGMP treatment did not reverse the EGF-induced phosphorylation of EGFR, MEK and ERK. (D) Co-immunoprecipitation was used to detect the effect of EGF and endogenous PKG on the binding between Grb2 and Sos1. Results showed that EGF treatment increased the binding level between Grb2 and Sos1. cGMP treatment did not inhibit the EGF-induced binding between Grb2 and Sos1. (E) Pull-down assay was performed to analyze the activated Ras. Results showed that EGF treatment caused a notable activation of Ras. cGMP treatment did not reverse EGF-induced Ras activation. detect the level of p-VASP Ser239 following treatment with cGMP in SCLC-239A cells. Results showed that increased endogenous PKG activity by cGMP treatment did not trigger the phosphorylation of VASP at Ser239 in SCLC-239A cells (Fig. 4B). Finally, we analyzed the role of p-VASP Ser239 in endogenous PKG activity in the EGF-induced MAPK/ERK signaling pathway. The SCLC-239A cells were applied and the effects of cGMP on EGF-induced MAPK/ERK signaling were measured. Western blotting was applied to detect the effect of endogenous PKG activity on the phosphorylation of EGFR (Y1068) in EGF-treated SCLC-239A cells. The results showed that treatment with cGMP did not inhibit the EGF-induced phosphorylation of EGFR (Y1068; Fig. 4C). In order to analyze the binding between Grb2 and Sos1 in SCLC-239A cells, co-immunoprecipitation assay was applied. Results showed that, compared with EGF treatment alone, there was no detectable alteration on this binding with the treatment of cGMP (Fig. 4D). Pull-down assay and western blotting were applied to detect the Ras activity in SCLC-239A cells. Results showed that cGMP treatment did not inhibit Ras activation induced by EGF (Fig. 4E). Furthermore, western blot analysis was applied to investigate the effects of cGMP on EGF-induced phosphorylation of MEK and ERK in SCLC-239A cells. Results showed that with the treatment of cGMP, there was no detectable decrease in MEK and ERK phosphorylation (Fig. 4F). Together, these data demonstrate that p-VASP Ser239 is critical for endogenous PKG activity in the EGF-induced MAPK/ERK signal transduction pathway.

\section{Discussion}

EGFR, a transmembrane tyrosine kinase, can be selectively activated through the binding with ligands belonging to the EGF family of peptide growth factors. Its mechanism of activation depends on the receptor dimerization and auto-phosphorylation. The adaptor proteins bind EGFR at phospho-Tyr1068 and phospho-Tyr1086 and activate the MAP kinase and PI3K/Akt pathways $(27,28)$. The majority of human epithelial cancers are marked by the activation of EGFR, and it was the first growth factor receptor to be proposed as a target for cancer therapy. Anti-EGFR therapies have been recently developed for the treatment of multiple cancer types.

PKG II is membrane-anchored, with high levels in brain and intestinal mucosa and low levels in certain human cancer cell lines (29). Previous research indicated that PKG II was associated with proliferation and apoptosis in certain cells $(30,31)$. Our previous study revealed that exogenous PKG II suppressed EGF-induced MAPK/ERK-mediated signal transduction in gastric cancer cells (18). However, the mechanisms involved remained unknown. In order to investigate its functions further, we focused on the endogenous PKG as well as exogenous PKG. In this study, we aimed to investigate the role of endogenous PKG on EGF-triggered MAPK/ERK signaling, and further focused on its possible mechanism in this signaling pathway.

In the present study, PKG-selective cGMP analog 8-pCPT-cGMP was applied to increase the endogenous PKG activity. Through confirming the effect of EGF on the MAPK/ERK signaling pathway, we analyzed the role of endogenous PKG on the EGF-triggered MAPK/ERK signal transduction pathway. Compared with the EGF treatment 
alone, 8-pCPT-cGMP treatment prior to EGF treatment notably blocked EGF-induced alteration, including the phosphorylation of EGFR, MEK and ERK, the binding between Sos1 and Grb2, and the Ras activity. Notably, with the treatment of cGMP followed by EGF, the phosphorylation of EGFR, MEK and ERK decreased, while the phosphorylation of VASP at Ser239 increased. VASP is a major substrate of PKG. In order to test the role of p-VASP Ser239 during this process, we designed lentivirus-mediated EGFP-VASP Ser239 site mutation (239A) to block its phosphorylation in SCLC cells. The results showed that the effects of endogenous PKG activity on EGF-induced MAPK/ERK signaling were notably attenuated. In SCLC-239A cells, with treatment of cGMP to increase the endogenous PKG activity, there was no increase in p-VASP Ser239, and compared with EGF treatment alone, increased endogenous PKG activity did not notably block the effect of EGF on MAPK/ERK signaling.

Our interpretation of the data presented in this study is that increased endogenous PKG activity inhibits the EGF-triggered MAPK/ERK signal transduction pathway, and p-VASP Ser239 plays a critical role. However, the exact role of p-VASP Ser239 in $\mathrm{PKG} / \mathrm{MAPK} / \mathrm{ERK}$ signal transduction requires further investigation.

\section{Acknowledgements}

This study was supported by the National Natural Science Foundation of China (Grant No. 31100974 and 81001100) and the Specialized Research Fund for Senior Personnel Program of Jiangsu University (Grant No. 11JDG032).

\section{References}

1. Wells A: EGF receptor. Int J Biochem Cell Biol 31: 637-643, 1999.

2. Wheeler DL, Dunn EF and Harari PM: Understanding resistance to EGFR inhibitors-impact on future treatment strategies. Nat Rev Clin Oncol 7: 493-507, 2010.

3. Hackel PO, Zwick E, Prenzel N and Ullrich A: Epidermal growth factor receptors: critical mediators of multiple receptor pathways. Curr Opin Cell Biol 11: 184-189, 1999.

4. Jiang H, Grenley MO, Bravo MJ, Blumhagen RZ and Edgar BA: EGFR/Ras/MAPK signaling mediates adult midgut epithelial homeostasis and regeneration in Drosophila. Cell Stem Cell 8: 84-95, 2011.

5. Yamazaki T, Zaal K, Hailey D, Presley J, Lippincott-Schwartz J and Samelson LE: Role of Grb2 in EGF-stimulated EGFR internalization. J Cell Sci 115: 1791-1802, 2002.

6. Downward J: Cell cycle: routine role for Ras. Curr Biol 7: 258-260, 1997

7. Kolch W: Meaningful relationships: the regulation of the Ras/Raf/MEK/ERK pathway by protein interactions. Biochem J 2: 289-305, 2000.

8. Stockand JD and Meszaros JG: Aldosterone stimulates proliferation of cardiac fibroblasts by activating Ki-RasA and MAPK1/2 signaling. Am J Physiol Heart Circ Physiol 284: H176-H184, 2003.

9. Martindale JL and Holbrook NJ: Cellular response to oxidative stress: signaling for suicide and survival. J Cell Physiol 192: 1-15, 2002.

10. Orstavik S, Natarajan V, Taskén K, Jahnsen T and Sandberg M: Characterization of the human gene encoding the type Ialpha and type Ibeta cGMP-dependent protein kinase (PRKG1). Genomics 42: 311-318, 1997.

11. Markert T, Vaandrager AB, Gambaryan S, Pöhler D, Häusler C, Walter U, De Jonge HR, Jarchau $\mathrm{T}$ and Lohmann SM: Endogenous expression of type II cGMP-dependent protein kinase mRNA and protein in rat intestine. Implications for cystic fibrosis transmembrane conductance regulator. J Clin Invest 96: $822-830,1995$
12. Haslam RJ, Dickinson NT and Jang EK: Cyclic nucleotides and phosphodiesterases in platelets. Thromb Haemost 82: 412-423, 1999.

13. Schlossmann J, Ammendola A, Ashman K, Zong X, Huber A, Neubauer G, Wang GX, Allescher HD, Korth M, Wilm M, Hofmann F and Ruth P: Regulation of intracellular calcium by a signalling complex of IRAG, IP3 receptor and cGMP kinase Ibeta. Nature 404: 197-201, 2000.

14. Ammendola A, Geiselhöringer A, Hofmann F and Schlossmann J: Molecular determinants of the interaction between the inositol 1,4,5-trisphosphate receptor-associated cGMP kinase substrate (IRAG) and cGMP kinase Ibeta. J Biol Chem 276: 24153-24159, 2001.

15. Russo I, Doronzo G, De Salve A, Mattiello L, Trovati M and Anfossi G: The activity of constitutive nitric oxide synthase is increased by the pathway cAMP/cAMP-activated protein kinase in human platelets. New insights into the antiaggregating effects of cAMP-elevating agents. Thromb Res 114: 265-273, 2004.

16. Swartling FJ, Ferletta M, Kastemar M, Weiss WA and Westermark B: Cyclic GMP-dependent protein kinase II inhibits cell proliferation, Sox9 expression and Akt phosphorylation in human glioma cell lines. Oncogene 28: 3121-3131, 2009.

17. Chen YC, Ren F, Sang JR, Tao Y and Xu WX: Type II cGMP-dependent protein kinase inhibits proliferation of the gastric cancer cell line BGC-823. Mol Med Report 3: 361-366, 2010.

18. Wu Y, Chen YC, Qu R, Lan T and Sang JR: Type II cGMP-dependent protein kinase inhibits EGF-triggered signal transduction of the MAPK/ERK-mediated pathway in gastric cancer cells. Oncol Rep 27: 553-558, 2012.

19. Geese M, Loureiro JJ, Bear JE, Wehland J, Gertler FB and Sechi AS: Contribution of Ena/VASP proteins to intracellular motility of listeria requires phosphorylation and proline-rich core but not F-actin binding or multimerization. Mol Biol Cell 13: 2383-2396, 2002.

20. Lois C, Hong EJ, Pease S, Brown EJ and Baltimore D: Germline transmission and tissue-specific expression of transgenes delivered by lentiviralvectors. Science 295: 868-872, 2002.

21. Zhang XY, La Russa VF and Reiser J: Transduction of bone-marrow-derived mesenchymal stem cells by using lentivirus vectors pseudotyped with modified RD114 envelope glycoproteins. J Virol 78: 1219-1229, 2004.

22. de Rooij J and Bos JL: Minimal Ras-binding domain of Raf1 can be used as an activation-specific probe for Ras. Oncogene 14: 623-625, 1997.

23. Draijer R, Vaandrager AB, Nolte C, de Jonge HR, Walter U and van Hinsbergh VW: Expression of cGMP-dependent protein kinase I and phosphorylation of its substrate, vasodilator-stimulated phosphoprotein, in human endothelial cells of different origin. Circ Res 77: 897-905, 1995.

24. Butt E, Abel K, Krieger M, Palm D, Hoppe V, Hoppe J and Walter U: cAMP- and cGMP-dependent protein kinase phosphorylation sites of the focal adhesion vasodilator-stimulated phosphoprotein (VASP) in vitro and in intact human platelets. J Biol Chem 269: 14509-14517, 1994.

25. Gertler FB, Niebuhr K, Reinhard M, Wehland J and Soriano P: Mena, a relative of VASP and Drosophila Enabled, is implicated in the control of microfilament dynamics. Cell 87: 227-239, 1996.

26. Lambrechts A, Kwiatkowski AV, Lanier LM, Bear JE, Vandekerckhove J, Ampe C and Gertler FB: cAMP-dependent protein kinase phosphorylation of EVL, a Mena/VASP relative, regulates its interaction with actin and SH3 domains. J Biol Chem 275: 36143-36151, 2000.

27. Li S, Couvillon AD, Brasher BB and Van Etten RA: Tyrosine phosphorylation of Grb2 by Bcr/Abl and epidermal growth factor receptor: a novel regulatory mechanism for tyrosine kinase signaling. EMBO J 20: 6793-6804, 2001.

28. Rodrigues GA, Falasca M, Zhang Z, Ong SH and Schlessinger J: A novel positive feedback loop mediated by the docking protein Gab1 and phosphatidylinositol 3-kinase in epidermal growth factor receptor signaling. Mol Cell Biol 20: 1448-1459, 2000.

29. Schlossmann J, Feil R and Hofmann F: Insights into cGMP signalling derived from cGMP kinase knockout mice. Front Biosci 10: 1279-1289, 2005.

30. Cook AL and Haynes JM: Protein kinase G II-mediated proliferative effects in human cultured prostatic stromal cells. Cell Signal 16: 253-261, 2004.

31. Cook AL and Haynes JM: Phosphorylation of the PKG substrate, vasodilator-stimulated phosphoprotein (VASP), in human cultured prostatic stromal cells. Nitric Oxide 16: 10-17, 2007. 